\title{
Prevalência e fatores associados ao diagnóstico de câncer de mama em estágio avançado
}

\author{
Prevalence and factors associated to advanced stage breast cancer \\ diagnosis
}

Tainá Bastos dos Santos (https://orcid.org/0000-0003-1365-6642) ${ }^{1}$

Anne Karin da Mota Borges (https://orcid.org/0000-0003-1322-3986) ${ }^{2}$

Jeniffer Dantas Ferreira (https://orcid.org/0000-0002-6724-5614) ${ }^{3}$

Karina Cardoso Meira (https://orcid.org/0000-0002-1722-5703) ${ }^{4}$

Mirian Carvalho de Souza (https://orcid.org/0000-0001-7516-1974) ${ }^{3}$

Raphael Mendonça Guimarães (https://orcid.org/0000-0003-1225-6719) ${ }^{5}$

Rafael Tavares Jomar (https://orcid.org/0000-0002-4101-7138) ${ }^{6}$

${ }^{1}$ Programa de Residência

Multiprofissional em

Oncologia, Instituto

Nacional de Câncer José

de Alencar Gomes da

Silva (INCA). Praça Cruz

Vermelha 23, Centro. 20230-

130 Rio de Janeiro RJ Brasil.

taina.bastos05@gmail.com

${ }^{2}$ Coordenação de Gestão de

Pessoas, Instituto Nacional

de Câncer José de Alencar

Gomes da Silva (INCA). Rio

de Janeiro RJ Brasil.

${ }^{3}$ Coordenação de Prevenção

e Vigilância, Instituto

Nacional de Câncer José

de Alencar Gomes da Silva

(INCA). Rio de Janeiro RJ

Brasil.

${ }^{4}$ Escola de Saúde,

Universidade Federal do Rio

Grande do Norte. Natal RN

Brasil.

${ }^{5}$ Escola Politécnica de

Saúde Joaquim Venâncio,

Fundação Oswaldo Cruz.

Rio de Janeiro RJ Brasil.

${ }^{6}$ Coordenação de

Assistência, Instituto

Nacional de Câncer José

de Alencar Gomes da Silva

(INCA). Rio de Janeiro RJ

Brasil.

\begin{abstract}
This cross-sectional study investigated the prevalence and factors associated with advanced stage breast cancer diagnosis among 18,890 women assisted in a specialized hospital in the capital of Rio de Janeiro, Brazil, from 1999 to 2016. We used Poisson regression with robust variance to estimate prevalence ratios and respective 95\% confidence intervals. The highest prevalence of diagnosis in this condition was in women aged 20-39 and 40-49 years, black and brown, living without a partner, from other cities of the state of Rio de Janeiro, who were referred by the Unified Health System and diagnosed in the 1999-2004 and 2005-2010 six-year periods. On the other hand, women aged 60-69 and 70-99 years, with some schooling level, with a family history of cancer and who arrived at the hospital with diagnosis and without treatment had lower prevalence of advanced stage breast cancer diagnosis. These results can be considered when planning secondary prevention actions to anticipate and, thus, collaborate to reduce the prevalence the breast cancer diagnosis in the most vulnerable groups.

Key words Breast neoplasms, Neoplasm staging, Cancer care facilities, Electronic health records, Cross-sectional studies
\end{abstract}

Resumo Este estudo transversal investigou a prevalência e os fatores associados ao diagnóstico de câncer de mama em estágio avançado entre 18.890 mulheres assistidas em hospital especializado da capital do Rio de Janeiro, Brasil, entre os anos 1999 e 2016. Utilizou-se regressão de Poisson com variância robusta para estimar razões de prevalência e respectivos intervalos de $95 \%$ de confiança. Apresentaram maiores prevalências de diagnóstico nessa condição mulheres com idade entre 20-39 e 40-49 anos, de raça/cor da pele preta e parda, que viviam sem companheiro(a), procedentes de outros municípios do estado do Rio de Janeiro, que tiveram o Sistema Único de Saúde como origem do encaminhamento e que foram diagnosticadas nos sexênios 1999-2004 e 20052010. Em contrapartida, mulheres com idade entre 60-69 e 70-99 anos, que cursaram algum nivel de escolaridade, com histórico familiar de câncer e que chegaram ao hospital com diagnóstico e sem tratamento apresentaram menores prevalências de diagnóstico em estágio avançado. Esses resultados podem ser considerados no planejamento de ações de prevenção secundária, a fim de antecipar o diagnóstico de câncer de mama dos grupos mais vulneráveis e assim colaborar para a redução da prevalência do diagnóstico em estágio avançado.

Palavras-chave Neoplasias da mama, Estadiamento de neoplasias, Institutos de câncer, Registros eletrônicos de saúde, Estudos transversais 


\section{Introdução}

O câncer de mama é a neoplasia mais comumente diagnosticada em mulheres, configurando-se em importante problema de saúde pública mundial devido à sua elevada incidência e morbimortalidade. Segundo o Observatório Global de Câncer, foram estimados 2,2 milhões de novos casos e 655 mil óbitos pela doença para $2020^{1}$.

No Brasil, o Instituto Nacional de Câncer José Alencar Gomes da Silva (INCA) estimou, para cada ano do triênio 2020-2022, 66.280 novos casos de câncer de mama. Excetuando-se os tumores de pele não melanoma, o câncer de mama será o mais incidente nas mulheres das regiões Sul (71,16/100 mil), Sudeste (81,06/100 mil), Centro-Oeste $(45,24 / 100$ mil) e Nordeste $(44,29 / 100$ mil); apenas na região Norte será o segundo mais incidente $(21,34 / 100 \mathrm{mil})^{2}$. As maiores taxas de incidência, portanto, serão observadas nas regiões de maior desenvolvimento socioeconômico do país. Cabe destacar também que o câncer de mama se configurou como a principal causa de morte por câncer entre as mulheres em 2017, ano em que houve 16.724 mortes por essa causa no Brasil (16,16/100 mil $)^{3}$.

Apesar de sua evolução temporal ascendente, o câncer de mama vem apresentando tendência descendente de mortalidade em países desenvolvidos $^{1}$. Acredita-se que isso esteja relacionado ao maior acesso a serviços de saúde, que possibilita o diagnóstico precoce e o tratamento oportuno da doença, aumentando assim a sobrevida ${ }^{1,3}$. Entretanto, a mortalidade no Brasil permanece elevada, sendo o diagnóstico tardio o principal motivo ${ }^{4,5}$.

No contexto nacional, convém citar dois estudos. O primeiro deles foi conduzido com 43.442 casos de câncer de mama atendidos entre os anos 1995 e 2002 pelo Sistema Único de Saúde (SUS): o diagnóstico em estágio avançado - III e IV representou $45 \%$ de todos os casos analisados ${ }^{6}$. O segundo foi realizado a partir de informações produzidas pelos registros hospitalares de câncer no país entre os anos 2000 e 2012, com 170.757 casos de câncer de mama, identificando tendência de aumento do diagnóstico em estágios III e IV no período 2002-2009 e estabilidade no período 2009-20127.

Em outro estudo, realizado em 2004, envolvendo 104 mulheres com câncer de mama assistidas em hospital de referência no Rio de Janeiro, o diagnóstico em estágio avançado representou $51 \%$ dos casos $^{8}$. Vale destacar que pesquisas mostram que o diagnóstico de câncer de mama em estágio avançado está associado à renda, pois cerca de 40-60\% dos estágios III e IV no momento do diagnóstico ocorrem em mulheres de baixa renda $^{8,9}$. Além da baixa renda ${ }^{9,10}$, outros fatores já foram apontados pela literatura como associados ao diagnóstico de câncer de mama em estágio avançado, tais como raça/cor da pele preta ou $\operatorname{parda}^{11,12}$, ser jovem ${ }^{13}$, não possuir parceiro(a), ter conhecimento limitado sobre cuidados com a mama, barreiras socioculturais como medo do diagnóstico, temor das consequências do tratamento, vergonha, noções equivocadas sobre etiologia ${ }^{14}$, dificuldades no acesso à atenção bási$\mathrm{ca}^{15,16}$, não atendimento por profissional de saúde especializado $^{17,18}$ e maior intervalo de tempo entre suspeita mamográfica e biópsia ${ }^{19,20}$.

Considerando que o conhecimento dos determinantes individuais e contextuais do diagnóstico de câncer de mama em estágio avançado é imprescindível para o planejamento de ações da atenção básica e de unidades de assistência de alta complexidade em oncologia com vistas a melhorar indicadores de diagnóstico e de tratamento em tempo hábil, especialmente os de grupos mais vulneráveis, o presente estudo teve como objetivo investigar a prevalência e os fatores associados ao diagnóstico de câncer de mama em estágio avançado entre mulheres assistidas em hospital especializado.

\section{Métodos}

Trata-se de um estudo transversal desenvolvido com dados disponibilizados pelo IntegradorRHC, um sistema web desenvolvido pelo Ministério da Saúde por meio do INCA para consolidação de dados sistematicamente coletados pelos Registros Hospitalares de Câncer (RHC) de todo o Brasil. A população-alvo do estudo foram os casos de câncer de mama feminina (código topográfico C50 da Classificação Internacional de Doenças - CID-10) $)^{21}$ cadastrados pelo RHC do Hospital do Câncer III/INCA e diagnosticados entre os anos 1999 e 2016. O Hospital do Câncer III/INCA, localizado na capital homônima do estado do Rio de Janeiro, Brasil, é especializado no tratamento do câncer de mama e presta assistência médico-hospitalar pelo SUS, sendo um centro de referência para a população do estado.

Foram elegíveis para o estudo mulheres com 20 anos de idade ou mais na data da primeira consulta médica, com diagnóstico de câncer de mama dos tipos carcinoma infiltrante (ductal ou lobular - códigos histológicos 8500/3 e 8520/3 da 
Classificação Internacional de Doenças para Oncologia - CID-O $)^{22}$ confirmado por exame histopatológico $(\mathrm{n}=21.171)$. Foram excluídos os casos sem informação de estadiamento clínico $(\mathrm{n}=$ $447 ; 2,11 \%)$ e os casos não analíticos $(n=2.025$; $9,56 \%$ ), isto é, aqueles diagnosticados e tratados em outras instituições e que foram ao Hospital do Câncer III/INCA apenas para complementar o tratamento antineoplásico, sem no entanto ter sua evolução acompanhada por essa unidade. Cabe destacar que a exclusão de casos não analíticos se deu porque a intenção deste estudo foi investigar somente os casos cujos tratamentos e consequente acompanhamento estiveram sob a responsabilidade do Hospital do Câncer III/ INCA, ou seja, os casos analíticos.

As variáveis incluídas no estudo foram categorizadas da seguinte forma: idade na primeira consulta (faixas etárias: 20-39, 40-49, 50-59, 6069 e 70-99 anos); raça/cor da pele (branca, preta, parda e amarela/indígena); escolaridade (nenhuma, ensino fundamental incompleto, ensino fundamental completo, ensino médio e ensino superior completo); situação conjugal (vive com companheiro/a e vive sem companheiro/a); município de procedência (capital do Rio de Janeiro, outros pertencentes ao Rio de Janeiro e outros não pertencentes ao Rio de Janeiro); histórico familiar de qualquer tipo de câncer (sim e não); origem do encaminhamento (SUS, não SUS e por conta própria); histórico de diagnóstico e de tratamento anteriores à chegada ao hospital (sem diagnóstico e sem tratamento, com diagnóstico e sem tratamento e com diagnóstico e com tratamento); período de diagnóstico (sexênios: 19992004, 2005-2010 e 2011-2016); tipo histológico do tumor (carcinoma ductal infiltrante e carcinoma lobular infiltrante); e estadiamento clínico do tumor (estágio não avançado, se I ou II, e estágio avançado, se III ou IV).

Quanto à categorização em sexênios da variável período de diagnóstico, é importante destacar que houve mudança no encaminhamento de usuárias para o Hospital do Câncer III/INCA a partir de 2011, primeiro ano do sexênio mais recente, no qual o encaminhamento passou a ser feito predominantemente por meio da Central de Regulação de Alta Complexidade do Rio de Janeiro ${ }^{23}$. Também é digno de nota que foram consideradas elegíveis para o estudo mulheres com 20 anos ou mais de idade porque casos de câncer com até 19 anos são considerados infanto-juvenis ${ }^{2}$.

$\mathrm{Na}$ análise de dados, realizada no software Stata, versão 15.0, a população de estudo foi descrita por meio de frequências absolutas e rela- tivas. A prevalência do diagnóstico de câncer de mama em estágio avançado - global e segundo características sociodemográficas, clínicas e relacionadas ao diagnóstico e ao tratamento - foi calculada com seus respectivos intervalos de 95\% de confiança (IC 95\%). Para investigar os fatores associados ao diagnóstico de câncer de mama em estágio avançado, foi utilizada a regressão de Poisson com variância robusta, que estimou razões de prevalência (RP) brutas e ajustadas e seus respectivos IC 95\%. Todas as variáveis que na análise univariada apresentaram $\mathrm{p} \leq 0,20$ no teste de Wald foram incluídas conjuntamente na análise multivariada. Vale sublinhar que, para calcular a prevalência e as RP, foram excluídos os casos com variáveis sem informação (dados faltantes).

Com relação aos aspectos éticos, convém mencionar que os dados disponibilizados pelo IntegradorRHC, além de não identificarem os casos de câncer, são de domínio público e de acesso irrestrito (https://irhc.inca.gov.br/RHCNet). Portanto, não foi necessária a apreciação do protocolo deste estudo por Comitê de Ética em Pesquisa com Seres Humanos, conforme preconizado pelo Conselho Nacional de Saúde.

\section{Resultados}

Atenderam aos critérios de elegibilidade do estudo 18.890 mulheres, com idade média de 56,1 anos (desvio-padrão $=13,3$ ). Aquelas com idade entre 50 e 59 anos representaram 27,19\% dos casos. Em relação à cor da pele, 57,65\% eram brancas. Quanto à escolaridade, 42,77\% tinham ensino fundamental incompleto. Pouco mais da metade $(53,36 \%)$ vivia sem companheiro(a). No que diz respeito ao município de procedência, 57,03\% eram da capital do Rio de Janeiro. Com relação ao histórico familiar de câncer, 57,27\% tinham pelo menos um familiar diagnosticado com a doença. O sexênio 1999-2004 apresentou a maior proporção de casos diagnosticados $(35,40 \%)$. A maioria das participantes foi encaminhada por um serviço vinculado ao SUS $(66,16 \%)$, e somente $1,40 \%$ chegou com diagnóstico e com tratamento, sendo classificados pelo RHC como casos analíticos porque não só o tratamento antineoplásico foi continuado no Hospital do Câncer III/INCA como sua evolução também foi por ele acompanhada. O tipo histológico mais frequente foi o carcinoma ductal infiltrante $(92,44 \%)$. Quanto ao estadiamento clínico, 39,16\% das mulheres foram diagnosticadas em estágio II e 33,23\% em estágio III (Tabela 1). 
Tabela 1. Características sociodemográficas, clínicas e relacionadas ao diagnóstico e ao tratamento da população de estudo. Hospital do Câncer III/Instituto Nacional de Câncer José Alencar Gomes da Silva, 1999-2016 (n = 18.890).

\begin{tabular}{|c|c|c|c|}
\hline Variáveis & n (\%) & Variáveis & n (\%) \\
\hline Faixa etária (em anos) & & Histórico familiar de câncer & \\
\hline $20-39$ & $1.933(10,23)$ & Não & $7.663(40,57)$ \\
\hline $40-49$ & $4.498(23,81)$ & Sim & $10.272(54,38)$ \\
\hline $50-59$ & $5.136(27,19)$ & Sem informação & $955(5,06)$ \\
\hline $60-69$ & $3.969(21,01)$ & Origem do encaminhamento & \\
\hline $70-99$ & $3.354(17,76)$ & Não SUS & $5.476(28,99)$ \\
\hline Raça/cor da pele & & SUS & $12.225(64,72)$ \\
\hline Branca & $10.844(57,41)$ & Por conta própria & $778(4,12)$ \\
\hline Preta & $2.975(15,75)$ & Sem informação & $411(2,18)$ \\
\hline Parda & $4.971(26,32)$ & Período de diagnóstico (em & \\
\hline Amarela/indígena & $21(0,11)$ & sexênios) & \\
\hline Sem informação & $79(0,42)$ & 1999-2004 & $6.687(35,40)$ \\
\hline Escolaridade & & $2005-2010$ & $6.536(34,60)$ \\
\hline Nenhuma & $1.041(5,51)$ & 2011-2016 & $6.667(30,00)$ \\
\hline Ensino fundamental incompleto & $8.051(42,62)$ & Diagnóstico e tratamento anteriores & \\
\hline Ensino fundamental completo & $3.362(17,80)$ & Sem diagnóstico e sem tratamento & $8.338(44,14)$ \\
\hline Ensino médio & $4.662(24,68)$ & Com diagnóstico e sem & $10.287(54,46)$ \\
\hline Ensino superior completo & $1.706(9,03)$ & tratamento & \\
\hline Sem informação & $68(0,36)$ & Com diagnóstico e com & $265(1,40)$ \\
\hline Situação conjugal & & tratamento & \\
\hline Vive com companheiro(a) & $8.712(46,12)$ & Tipo histológico do tumor & \\
\hline Vive sem companheiro(a) & $9.966(52,76)$ & Carcinoma ductal infiltrante & $17.461(92,44)$ \\
\hline Sem informação & $212(1,12)$ & Carcinoma lobular infiltrante & $1.429(7,56)$ \\
\hline Município de procedência & & Estadiamento clínico do tumor & \\
\hline Capital do Rio de Janeiro & $10.772(57,02)$ & I & $3.243(17,17)$ \\
\hline Outros pertencentes ao Rio de & $8.036(42,54)$ & II & $7.397(39,16)$ \\
\hline Janeiro & & III & $6.278(33,23)$ \\
\hline \multirow{2}{*}{$\begin{array}{l}\text { Outros não pertencentes ao Rio } \\
\text { de Janeiro }\end{array}$} & \multirow[t]{2}{*}{$80(0,42)$} & IV & $1.972(10,44)$ \\
\hline & & onte: IntegradorRHC. & \\
\hline Sem informação & $02(0,01)$ & & \\
\hline
\end{tabular}

A prevalência global do diagnóstico de câncer de mama em estágio avançado (estadiamento clínico III e IV) foi de 43,67\% (IC 95\%: 42,9644,38) (Tabela 2). No modelo de regressão multivariada, observou-se que, quando comparadas às mulheres com idade entre 50 e 59 anos, aquelas de 20 a 39 e de 40 a 49 tiveram maiores prevalências de diagnóstico em estágio avançado (respectivamente, RP = 1,31; IC 95\%: 1,24-1,38; e RP = 1,08; IC 95\%: 1,03-1,13), e aquelas com idade entre 60 e 69 e entre 70 e 99 anos apresentaram menor prevalência de diagnóstico nessa condição (respectivamente, RP = 0,85; IC 95\%: 0,81-0,90; e RP = 0,88; IC 95\%: 0,83-0,93). Mulheres pretas, quando comparadas às brancas, também tiveram maior prevalência de diagnóstico de câncer de mama em estágio avançado ( $\mathrm{RP}=1,22$; IC 95\%: $1,17-1,28)$, tal como as pardas $(\mathrm{RP}=1,13$; IC 95\%: 1,09-1,18) (Tabela 3).

No que se refere à escolaridade, quando comparadas àquelas sem nenhuma, foi observado um gradiente de forma que, quanto maior a escolaridade, menor a prevalência de diagnóstico de câncer de mama em estágio avançado. Mulheres que viviam sem companheiro(a) apresentaram maior prevalência de diagnóstico em estágio avançado (RP = 1,09; IC 95\%: 1,05-1,13) quando comparadas às que viviam com companheiro(a) (Tabela 3).

Quanto à cidade de procedência, mulheres oriundas de outros municípios pertencentes ao Rio de Janeiro apresentaram maior prevalência 
Tabela 2. Prevalência e respectivos intervalos de 95\% de confiança (IC 95\%) do diagnóstico de câncer de mama em estágio avançado segundo características sociodemográficas, clínicas e relacionadas ao diagnóstico e ao tratamento. Hospital do Câncer III/Instituto Nacional de Câncer José Alencar Gomes da Silva, 1999-2016 (n = 18.890).

\begin{tabular}{|c|c|c|}
\hline \multirow{2}{*}{ Variáveis } & \multicolumn{2}{|c|}{ Diagnóstico em estágio avançado } \\
\hline & Não (IC 95\%) & Sim (IC 95\%) \\
\hline Global & $56,32(55,61-57,03)$ & $43,67(42,96-44,38)$ \\
\hline \multicolumn{3}{|l|}{ Faixa etária (em anos) } \\
\hline $20-39$ & $45,73(43,52-47,96)$ & $54,26(52,03-56,47)$ \\
\hline $40-49$ & $53,84(52,38-55,29)$ & $46,15(44,70-47,61)$ \\
\hline $50-59$ & $57,10(55,74-58,45)$ & $42,89(41,54-44,25)$ \\
\hline $60-69$ & $61,70(60,17-63,20)$ & $38,29(36,79-39,82)$ \\
\hline $70-99$ & $58,19(56,52-59,85)$ & $41,80(40,14-43,47)$ \\
\hline \multicolumn{3}{|l|}{ Raça/cor da pele } \\
\hline Branca & $60,38(59,45-61,30)$ & $39,61(38,69-40,64)$ \\
\hline Preta & $46,75(44,96-48,55)$ & $53,24(51,44-55,03)$ \\
\hline Parda & $53,10(51,71-54,49)$ & $46,89(45,50-48,28)$ \\
\hline Amarela/indígena & $57,14(35,97-75,98)$ & $42,85(24,01-64,02)$ \\
\hline \multicolumn{3}{|l|}{ Escolaridade } \\
\hline Nenhuma & $44,86(41,86-47,89)$ & $55,13(52,10-58,13)$ \\
\hline Ensino fundamental incompleto & $53,63(52,54-54,72)$ & $46,36(45,27-47,45)$ \\
\hline Ensino fundamental completo & $56,63(54,95-58,30)$ & $43,36(41,69-45,04)$ \\
\hline Ensino médio & $59,78(58,36-61,18)$ & $40,21(38,81-41,63)$ \\
\hline Ensino superior completo & $65,76(63,48-67,98)$ & $34,23(32,01-36,51)$ \\
\hline \multicolumn{3}{|l|}{ Situação conjugal } \\
\hline Vive com companheiro(a) & $57,98(56,94-59,02)$ & $42,01(40,97-43,05)$ \\
\hline Vive sem companheiro(a) & $54,78(53,80-55,76)$ & $45,21(44,23-46,19)$ \\
\hline \multicolumn{3}{|l|}{ Município de procedência } \\
\hline Capital do Rio de Janeiro & $58,34(57,41-59,27)$ & $41,65(40,72-42,58)$ \\
\hline Outros pertencentes ao Rio de Janeiro & $53,60(52,51-54,69)$ & $46,39(45,30-47,48)$ \\
\hline Outros não pertencentes ao Rio de Janeiro & $56,25(45,25-66,66)$ & $43,75(33,33-54,74)$ \\
\hline \multicolumn{3}{|l|}{ Histórico familiar de câncer } \\
\hline Não & $52,13(51,01-53,25)$ & $47,86(46,74-48,98)$ \\
\hline Sim & $60,31(59,36-61,26)$ & $39,68(38,73-40,63)$ \\
\hline \multicolumn{3}{|l|}{ Origem do encaminhamento } \\
\hline Não SUS & $60,31(59,01-61,60)$ & $39,68(38,39-40,98)$ \\
\hline SUS & $54,36(53,47-55,24)$ & $45,63(44,75-46,52)$ \\
\hline Por conta própria & $56,55(53,04-60,00)$ & $43,44(39,99-46,95)$ \\
\hline \multicolumn{3}{|l|}{ Período de diagnóstico (em sexênios) } \\
\hline $1999-2004$ & $53,16(51,96-54,35)$ & $46,83(45,64-48,03)$ \\
\hline $2005-2010$ & $56,60(55,40-57,80)$ & $43,39(42,19-48,03)$ \\
\hline 2011-2016 & $59,73(58,44-61,00)$ & $40,26(38,99-41,55)$ \\
\hline \multicolumn{3}{|l|}{ Diagnóstico e tratamento anteriores } \\
\hline Sem diagnóstico e sem tratamento & $53,94(52,87-55,01)$ & $46,05(44,98-47,12)$ \\
\hline Com diagnóstico e sem tratamento & $58,24(57,29-59,19)$ & $41,75(40,80-42,70)$ \\
\hline Com diagnóstico e com tratamento & $56,60(50,56-62,44)$ & $43,39(37,55-49,43)$ \\
\hline \multicolumn{3}{|l|}{ Tipo histológico do tumor } \\
\hline Carcinoma ductal infiltrante & $56,35(55,61-57,08)$ & $43,64(42,91-44,38)$ \\
\hline Carcinoma lobular infiltrante & $55,98(53,39-58,53)$ & $41,46(41,46-46,60)$ \\
\hline
\end{tabular}

Fonte: IntegradorRHC.

de diagnóstico de câncer de mama em estágio avançado (RP = 1,04; IC 95\%: 1,01-1,08) em comparação às que residiam na capital do estado. Mulheres que tinham como origem do encami- 
Tabela 3. Razões de prevalência (RP) brutas e ajustadas e respectivos intervalos de $95 \%$ de confiança (IC 95\%) do diagnóstico de câncer de mama em estágio avançado. Hospital do Câncer III/Instituto Nacional de Câncer José Alencar Gomes da Silva, 1999-2016.

\begin{tabular}{|c|c|c|c|}
\hline Variáveis & RP bruta (IC 95\%) & $\mathbf{p}^{*}$ & RP ajustada (IC 95\%) ${ }^{* *}$ \\
\hline Faixa etária (em anos) & & $<0,01$ & \\
\hline $50-59$ & 1,00 & & 1,00 \\
\hline $20-39$ & $1,26(1,20-1,33)$ & & $1,31(1,24-1,38)$ \\
\hline $40-49$ & $1,07(1,02-1,12)$ & & $1,08(1,03-1,13)$ \\
\hline $60-69$ & $0,89(0,84-0,93)$ & & $0,85(0,81-0,90)$ \\
\hline $70-99$ & $0,97(0,92-1,02)$ & & $0,88(0,83-0,93)$ \\
\hline Raça/cor da pele & & $<0,01$ & \\
\hline Branca & 1,00 & & 1,00 \\
\hline Preta & $1,34(1,29-1,40)$ & & $1,22(1,17-1,28)$ \\
\hline Parda & $1,18(1,13-1,2)$ & & $1,13(1,09-1,18)$ \\
\hline Amarela/indígena & $1,08(0,65-1,77)$ & & $1,20(0,70-2,05)$ \\
\hline Escolaridade & & $<0,01$ & \\
\hline Nenhuma & 1,00 & & 1,00 \\
\hline Ensino fundamental incompleto & $0,84(0,79-0,89)$ & & $0,83(0,78-0,89)$ \\
\hline Ensino fundamental completo & $0,78(0,73-0,84)$ & & $0,79(0,73-0,84)$ \\
\hline Ensino médio & $0,72(0,68-0,77)$ & & $0,72(0,67-0,78)$ \\
\hline Ensino superior completo & $0,62(0,56-0,67)$ & & $0,63(0,58-0,70)$ \\
\hline Situação conjugal & & $<0,01$ & \\
\hline Vive com companheiro(a) & 1,00 & & 1,00 \\
\hline Vive sem companheiro(a) & $1,07(1,04-1,11)$ & & $1,09(1,05-1,13)$ \\
\hline Município de procedência & & $<0,01$ & \\
\hline Capital do Rio de Janeiro & 1,00 & & 1,00 \\
\hline Outros pertencentes ao Rio de Janeiro & $1,11(1,07-1,15)$ & & $1,04(1,01-1,08)$ \\
\hline Outros não pertencentes ao Rio de Janeiro & $1,05(0,81-1,34)$ & & $1,05(0,80-1,37)$ \\
\hline Histórico familiar de câncer & & $<0,01$ & \\
\hline Não & 1,00 & & 1,00 \\
\hline $\operatorname{Sim}$ & $0,82(0,80-0,85)$ & & $0,84(0,80-0,89)$ \\
\hline Origem do encaminhamento & & $<0,01$ & \\
\hline Não SUS & 1,00 & & 1,00 \\
\hline SUS & $1,15(1,10-1,19)$ & & $1,09(1,05-1,14)$ \\
\hline Por conta própria & $1,09(1,01-1,19)$ & & $0,99(0,90-1,09)$ \\
\hline Período de diagnóstico (em sexênios) & & $<0,01$ & \\
\hline 2011-2016 & 1,00 & & 1,00 \\
\hline $2005-2010$ & $1,07(1,03-1,12)$ & & $1,09(1,04-1,14)$ \\
\hline 1999-2004 & $1,16(1,11-1,21)$ & & $1,10(1,05-1,15)$ \\
\hline Diagnóstico e tratamento anteriores & & $<0,01$ & \\
\hline Sem diagnóstico e sem tratamento & 1,00 & & 1,00 \\
\hline Com diagnóstico e sem tratamento & $0,90(0,87-0,93)$ & & $0,95(0,92-0,99)$ \\
\hline Com diagnóstico e com tratamento & $0,94(0,81-1,08)$ & & $0,93(0,72-1,09)$ \\
\hline Tipo histológico do tumor & & 0,78 & \\
\hline Carcinoma ductal infiltrante & 1,00 & & - \\
\hline Carcinoma lobular infiltrante & $1,01(0,94-1,07)$ & & - \\
\hline
\end{tabular}

${ }^{*}$ p-valor no teste de Wald. ${ }^{\star} \mathrm{RP}$ ajustada por todas as variáveis da tabela, exceto tipo histológico do tumor.

Fonte: IntegradorRHC.

nhamento o SUS também apresentaram maior prevalência de diagnóstico em estágio avançado ( $\mathrm{RP}=1,09$; IC 95\%: 1,05-1,14) quando comparadas às que não tinham sido encaminhadas pelo SUS. Aquelas diagnosticadas nos sexênios 1999-2004 e 2005-2010, isto é, em períodos anteriores ao encaminhamento de pacientes ser feito majoritariamente pela Central de Regulação de 
Alta Complexidade do Rio de Janeiro ${ }^{23}$, também apresentaram maiores prevalências de câncer de mama em estágio avançado (respectivamente, $\mathrm{RP}=1,10$; IC 95\%: 1,05-1,15; e RP = 1,09; IC 95\%: 1,04-1,14). Já as que chegaram ao hospital com diagnóstico e sem tratamento apresentaram menor prevalência de diagnóstico nessa condição $(\mathrm{RP}=0,95$; IC 95\%: 0,92-0,99) quando comparadas àquelas que chegaram sem diagnóstico e sem tratamento (Tabela 3 ).

\section{Discussão}

Os resultados do presente estudo mostram que a prevalência global do diagnóstico de câncer de mama em estágio avançado foi de $43,67 \%$ e que maiores prevalências de diagnóstico nessa condição foram observadas entre as mulheres com 49 anos de idade ou menos, de raça/cor da pele preta e parda, que viviam sem companheiro(a), procedentes de outros municípios pertencentes ao estado do Rio de Janeiro, que tiveram o SUS como origem do encaminhamento e que foram diagnosticadas antes de 2011. Em contrapartida, mulheres com idade maior ou igual a 60 anos, que cursaram algum nível de escolaridade, com histórico familiar de câncer e que chegaram ao hospital com diagnóstico e sem tratamento apresentaram menores prevalências de diagnóstico em estágio avançado.

No estudo, quando comparadas às mulheres com idade entre 50 e 59 anos, aquelas de 20 a 49 apresentaram maior prevalência de diagnóstico em estágio avançado. Uma investigação transversal conduzida entre 2000 e 2009 a partir de dados disponibilizados pelo IntegradorRHC relativos a 59.317 mulheres com câncer de mama de todo o Brasil ${ }^{24}$ encontrou resultados semelhantes aos nossos. Ratificando esses achados, outra investigação transversal realizada a partir de dados disponibilizados por registros de câncer de base populacional de dez estados norte-americanos, com 161.619 mulheres diagnosticadas com câncer de mama invasivo entre 2004 e 2006, apontou que o diagnóstico em estágio avançado foi maior entre aquelas com idade entre 40 e 49 anos $^{25}$.

Em consonância com os achados deste estudo, a literatura aponta que o risco de diagnóstico de câncer de mama em estágio avançado diminui com o aumento da idade. Um estudo transversal que investigou o câncer de mama localmente avançados a partir de dados do ano de 2006 do Surveillance, Epidemiology, and End Results, fonte oficial de informações para vigi- lância do câncer nos Estados Unidos, encontrou maior ocorrência desses casos entre mulheres jovens $^{26}$. O prognóstico para o câncer de mama em mulheres jovens é pior quando comparado ao de mulheres na pós-menopausa. Tal fato provavelmente se deve à maior prevalência de tumores mais agressivos nas jovens ${ }^{24}$.

Mulheres de raça/cor da pele preta e parda apresentaram maiores prevalências de diagnóstico em estágio avançado neste estudo. Em pesquisa transversal realizada a partir de dados disponibilizados pelo IntegradorRHC entre $2001 \mathrm{e}$ 2014 de 247.719 mulheres de todo o Brasil com câncer de mama invasivo, a prevalência de estágio avançado quando feito o diagnóstico também foi mais elevada entre aquelas de raça/cor da pele preta ou parda ${ }^{27}$. Um estudo longitudinal realizado a partir de dados dos anos de 2012 e 2013 do National Cancer Registration and Analysis Service, instituição responsável pelo registro de câncer na Inglaterra, com 86.852 casos de câncer de mama feminina apontou disparidades étnico -raciais, com maiores prevalências de diagnóstico em estágio avançado entre as mulheres de raça/ cor da pele preta ou parda ${ }^{28}$, corroborando os achados deste estudo.

Acredita-se que a raça/cor da pele seja uma variável proxy da dificuldade de acesso a bens, serviços, recursos sociais e de saúde da população negra feminina ${ }^{29}$. Tal fato decorre das iniquidades raciais em saúde, ligadas ao risco de adoecer e de morrer $^{30}$. Desigualdades em indicadores de saúde, juntamente com outros indicadores de posição social, como educação e renda, evidenciam a raça/cor de pele como um determinante social da pior saúde da população negra em comparação à branca ${ }^{31}$. Portanto, o estudo em questão parece corroborar essa afirmativa, uma vez que a raça/cor de pele preta e parda se mostrou associada ao diagnóstico de câncer de mama em estágio avançado.

Convém mencionar a Política Nacional de Saúde Integral da População Negra, instituída no Brasil em 2009, que, por meio do princípio da equidade, assegura o direito humano à saúde, com ações de cuidado, atenção, promoção, prevenção, tratamento e recuperação de doenças e agravos à saúde nesse segmento populacional ${ }^{32}$. Logo, essa política deve ser levada em conta no planejamento de ações de diagnóstico e de tratamento do câncer de mama em tempo hábil, a fim de melhor atender este grupo populacional vulnerável.

Em relação à escolaridade, quando comparadas àquelas sem nenhuma, mulheres com en- 
sino fundamental incompleto mostraram uma prevalência $17 \%$ menor de diagnóstico de câncer de mama em estágio avançado, sendo observado gradiente de aumento nessa estimativa quanto maior era a escolaridade. Estudo transversal feito a partir de dados disponibilizados pelo IntegradorRHC entre 2000 e 2009 com 59.317 casos de câncer de mama feminina de todo o Brasil apontou que mulheres com sete anos ou menos de estudo apresentaram chance $40 \%$ maior de serem diagnosticadas com câncer de mama em estágio avançado ${ }^{24}$. A educação - considerada uma proxy da situação socioeconômica - é um fator determinante das condições de saúde dos indivíduos, pois baixos níveis de escolaridade estão associados a piores condições de saúde ${ }^{33}$, conforme mostram os resultados do presente estudo. O conhecimento sobre a doença e as formas de detecção precoce, além do adequado acesso aos serviços de saúde, podem ser apontados, portanto, como determinantes de proteção contra o diagnóstico de câncer de mama em estágio avançado em mulheres com níveis de escolaridade mais altos.

No que diz respeito ao município de procedência, mulheres oriundas de outros que não os pertencentes ao Rio de Janeiro apresentaram maior prevalência de diagnóstico de câncer de mama em estágio avançado, em comparação com as que residiam na capital do estado. Uma hipótese para explicar tal achado é que esses municípios devem encaminhar os casos em estágios mais avançados para o Hospital do Câncer III/ INCA, responsabilizando-se apenas pelo tratamento daqueles diagnosticados em estágios iniciais. De modo geral, esses outros municípios não possuem dinamismo econômico suficiente, além de serem carentes em questões de saúde, educação, saneamento e emprego; alguns deles são até caracterizados como cidades dormitórios ${ }^{34}$. Um estudo de revisão sobre os fatores associados ao diagnóstico do câncer de mama em estágio avançado mostrou que residir em áreas de menor nível socioeconômico está significativamente associado ao diagnóstico tardio de câncer de mama ${ }^{13}$. Além disso, a distância geográfica de áreas menos abastadas e pobres para os grandes centros urbanos representam barreiras físicas, geográficas e socioculturais de acesso aos serviços de saúde ${ }^{15}$, que por sua vez é um sério determinante social de saúde.

As mulheres que viviam sem companheiro(a) apresentaram maior prevalência de diagnóstico em estágio avançado, quando comparadas às que viviam com companheiro(a). Em consonância com esses achados, um estudo caso-contro- le realizado com 540 casos hospitalares de câncer de mama nos Estados Unidos revelou que o risco de apresentar câncer em estágio avançado foi quase três vezes maior entre as mulheres que nunca foram casadas ${ }^{35}$. Resultados de um estudo longitudinal feito com dados disponibilizados pelo Surveillance, Epidemiology, and End Results envolvendo 32.268 mulheres diagnosticadas com câncer de mama entre 1991 e 1995 também nos EUA revelaram que as solteiras eram mais propensas ao diagnóstico em estágio avançado, apontando o maior apoio social e a melhor rede social como um privilégio das casadas ${ }^{36}$. Outro pesquisa longitudinal, desenvolvida a partir de dados de mulheres diagnosticadas com câncer de mama de 2005 a 2012 disponibilizados pelo $\mathrm{Ca}$ lifornia Cancer Registry (Estados Unidos) mostrou que as solteiras tinham maior taxa de mortalidade por câncer de mama dos que as casadas e apontou dois possíveis benefícios desta situação conjugal: melhores recursos econômicos e maior apoio social ${ }^{37}$.

De modo controverso, em um estudo de sobrevida envolvendo 1.022 mulheres com câncer de mama em estágio inicial, a situação conjugal não foi considerada fator determinante para o óbito $^{38}$. Por sua vez, uma revisão sistemática que avaliou os fatores de risco para o atraso no diagnóstico de câncer de mama encontrou evidências de que a situação conjugal não está relacionada ao diagnóstico em estágio avançado ${ }^{39}$. Duas razões são apontadas para os resultados desses estudos serem diferentes dos encontrados pelo presente: o estudo de sobrevida avaliou apenas casos em estágio não avançado, e a revisão sistemática não tratou a situação conjugal como uma variável proxy da rede de apoio social.

Os laços conjugais aumentam o tamanho da rede social não apenas por meio da disponibilidade de um parceiro(a), mas pelo acesso aos laços da rede dele $(a)^{37}$. Autores afirmam que os(as) parceiros(as) costumam promover comportamentos de saúde positivos, como incentivar visitas regulares a serviços de saúde ${ }^{40,41}$, o que favorece o diagnóstico precoce de doenças, inclusive do câncer de mama.

No que diz respeito ao apoio social, pesquisas apontam melhor sobrevida em mulheres com câncer de mama entre as com mais laços e maior apoio sociais ${ }^{42-46}$. Ainda nesse contexto, mulheres em estágio avançado decorrente do diagnóstico tardio possuem menos possibilidades de tratamentos curativos, o que demandaria maiores custos e cuidados com a saúde física e mental. Considerando-se as diferentes demandas e ne- 
cessidade de apoio, as solteiras ou aquelas sem rede de apoio poderiam, nesses casos, estar em desvantagem diante das casadas ${ }^{37,47}$.

Nossos resultados referentes à situação conjugal - aqui considerada uma variável proxy da rede de apoio social - devem ser analisados, portanto, à luz das controvérsias da literatura. Embora seja possível argumentar que mulheres sem apoio social vivenciam mais preocupações e problemas envolvendo decisões e cuidados com a saúde do que aquelas com apoio, os profissionais de saúde, diante do contexto de enfrentamento ao câncer de mama, devem, por meio de abordagem multiprofissional, enfatizar o acolhimento, o fortalecimento de vínculo e o aprimoramento das habilidades de enfrentamento, fornecendo apoio emocional e ampliando oportunidades de compartilhamento de informações tanto entre as solteiras quanto entre as casadas ${ }^{36,37,45}$.

A literatura aponta que a nuliparidade é um dos fatores de risco para o câncer de mama ${ }^{3,48}$, embora não seja um fator determinante. Do mesmo modo, ter laços conjugais não determina a vida reprodutiva da mulher, levando-a a ser mãe apenas porque vive ou viveu acompanhada. Especialmente na sociedade contemporânea, onde muito tem se argumentado a favor do empoderamento e do protagonismo feminino, do controle do próprio corpo e do direito de decidir sobre como e quando ter filhos ${ }^{49}$.

No que concerne ao histórico familiar de câncer, mulheres que possuíam história pregressa de qualquer tipo de câncer na família - e não somente de mama, conforme coleta sistemática dessa informação pelo RHC - apresentaram menor prevalência de diagnóstico em estágio avançado. A ausência de história familiar de câncer de mama, especificamente, já foi destacada pela literatura como importante fator associado ao diagnóstico $\operatorname{tardio}^{50}$. Então, pode-se especular que mulheres sem histórico familiar de câncer não se atentariam tanto e tão precocemente para a saúde das mamas quanto àquelas com história familiar. Em contrapartida, mulheres com esse antecedente, tendo algum conhecimento sobre a história natural da doença, sinais, sintomas, gravidade e potencial desfecho, além da maior demanda por mamografia ${ }^{51}$, estariam mais vigilantes à saúde das mamas e buscariam por serviços de saúde tão logo uma anormalidade fosse detectada no autoexame.

No que diz respeito aos fatores apontados na literatura que podem influenciar o atraso do diagnóstico, destacam-se o tempo até a busca por serviço de saúde após a suspeita inicial, a espera entre as consultas e a realização de exames, a morosidade de exames diagnósticos específicos e de encaminhamentos para especialistas, o tempo de espera entre o diagnóstico e início do tratamento, além de dificuldades envolvendo a referência e a contrarreferência nos serviços públicos de saú$\mathrm{de}^{11}$. Nesse sentido, estudos apontam que mulheres atendidas em hospitais públicos têm chance quase duas vezes maior de estágio avançado no momento do diagnóstico do câncer de mama ${ }^{52-54}$.

As dificuldades de acesso enfrentadas pela população economicamente desfavorecida que depende do SUS - retrato das desigualdades sociais em saúde no Brasil, embora o SUS seja um sistema de acesso universal - são apontadas como fator de risco para o desenvolvimento de doenças e o diagnóstico e tratamento ${ }^{55-57}$. Essas conclusões corroboram os resultados do presente estudo, em que mulheres cuja origem do encaminhamento foi o SUS tiveram prevalência de diagnóstico de câncer de mama em estágio avançado maior do que aquelas encaminhadas por serviços não vinculados ao SUS. Portanto, é importante também considerar a hipótese de que os casos diagnosticados em estágio avançado por serviços vinculados ao SUS sejam encaminhados ao Hospital do Câncer III/INCA porque ele é um centro de referência no Rio de Janeiro.

Os casos de câncer de mama que chegaram ao cenário de estudo com diagnóstico e sem tratamento apresentaram menor prevalência de diagnóstico em estágio avançado, da ordem de $5 \%$, em comparação com aqueles que chegaram sem diagnóstico e sem tratamento. Isso pode ter se dado porque o Hospital do Câncer III/INCA é um centro de alta complexidade em oncologia, referência no estado do Rio de Janeiro. Assim, para ele costumam ser encaminhados majoritariamente os casos de câncer de mama que terão mais opções de tratamento curativo, isto é, os casos diagnosticados em estágios iniciais; ainda que também receba casos em estágios mais avançados da doença oriundos de outros municípios do estado. Ratificam essa afirmativa os resultados acerca dos períodos de diagnóstico anteriores à formalização do encaminhamento de casos de câncer ser feita predominantemente pela Central de Regulação de Alta Complexidade do Rio de Janeiro, uma vez que esses períodos apresentaram maiores prevalências de diagnóstico em estágio avançado, o que talvez sugira maior agilidade no encaminhamento dos casos em estágio não avançado entre 2011 e 2016. Todavia, outros estudos devem ser conduzidos para esclarecer se o advento da Central de Regulação de Alta Comple- 
xidade do Rio de Janeiro está associado à maior agilidade no encaminhamento de mulheres com câncer de mama para hospitais especializados. Cabe ressaltar, por fim, que a mudança no encaminhamento de pacientes para o Hospital do Câncer III/INCA - considerada na etapa analítica do estudo - não modificou a direção nem a magnitude das associações encontradas, o que aponta para a inexistência de diferenças substanciais nas características sociodemográficas das pacientes admitidas antes e depois de 2011.

Como limitação do estudo, destaca-se a utilização de dados secundários que não possibilitaram a avaliação da influência de importantes variáveis no diagnóstico de câncer de mama em estágio avançado, como submissão a mamografia anterior ao diagnóstico e tempo decorrido entre os primeiros sintomas e a primeira consulta médica e/ou diagnóstico ${ }^{58}$, uma vez que os RHC do Brasil não coletam tais informações sistematicamente. Outra limitação que a ser mencionada é a morosa alimentação do IntegradorRHC, que, ao dificultar sua atualização com dados de anos mais recentes, impossibilitou a análise desses pelo estudo. Além disso, o Hospital do Câncer III/INCA é vinculado ao SUS, o que permite que seus resultados possam ser generalizados apenas para hospitais também vinculados ao SUS que prestam assistência a populações semelhantes.
Em face da ausência de informação em algumas variáveis (inferior a $5 \%$, vale enfatizar), não é possível ignorar o fato de que as medidas de associação estimadas poderiam ser ligeiramente maiores e os respectivos IC 95\% mais estreitos se o estudo tivesse realizado imputação múltipla de dados faltantes ${ }^{59}$. Por fim, é prudente considerar a ocorrência de viés de admissão no estudo, o que pode ter afetado a magnitude das associações encontradas, mas não a direção delas, uma vez que estão em consonância com a literatura.

Como ponto forte do estudo, sublinha-se o grande número de casos incluídos, bem como sua seleção rigorosa, o que garantiu a exclusão daqueles que não foram diagnosticados, tratados e acompanhados pelo cenário de investigação. Além disso, o estudo reforça o IntegradorRHC como importante fonte de pesquisa em saúde pública, uma vez que tal sistema se mostra útil para subsidiar o planejamento de ações de controle do câncer e consequentes aprimoramentos.

Em suma, acredita-se que os resultados deste estudo, ainda que limitados por seu desenho, que não permite inferência causal, devem ser considerados pelo Hospital do Câncer III/INCA no planejamento de suas ações de diagnóstico e de tratamento em tempo hábil, a fim de melhor assistir os subgrupos mais vulneráveis ao diagnóstico em estágio avançado de câncer de mama.

\section{Colaboradores}

TB Santos contribuiu na interpretação dos resultados, redação e revisão crítica relevante do conteúdo do artigo, além de acompanhar todas as etapas do trabalho na garantia da exatidão e integridade de qualquer parte da obra. JF Dantas, AKM Borges, KC Meira, MC Souza e RM Guimarães contribuíram na redação e revisão crítica relevante do conteúdo do artigo, além de acompanharem todas as etapas do trabalho na garantia da exatidão e integridade de qualquer parte da obra. RT Jomar contribuiu com a concepção do projeto, análise de dados, interpretação dos resultados, redação e revisão crítica relevante do conteúdo do artigo, além de acompanhar todas as etapas do trabalho na garantia da exatidão e integridade de qualquer parte da obra. 


\section{Referências}

1. Ferlay J, Ervik M, Lam F, Colombet M, Mery L, Piñe$\operatorname{ros}$ M, Znaor A, Soerjomataram I, Bray F. Global Cancer Observatory: Cancer Today. [Internet] Lyon: International Agency for Research on Cancer; 2018. [cited 2019 Aug 3]. Available from: https://gco.iarc.fr/today.

2. Instituto Nacional de Câncer José Alencar Gomes da Silva (INCA). Estimativa 2020: incidência de câncer no Brasil. Rio de Janeiro: INCA; 2019.

3. Instituto Nacional de Câncer José Alencar Gomes da Silva (INCA). A situação do câncer de mama no Brasil: sintese de dados dos sistemas de informação. Rio de Janeiro: INCA; 2019.

4. Oshiro ML, Bergmann A, Silva RG, Costa KC, Travaim IEB, Silva GB, Thuler LCS. Câncer de mama avançado como evento sentinela para avaliação do programa de detecção precoce do câncer de mama no Centro-Oeste do Brasil. Rev Bras Cancerol 2014; 60(1):15-23.

5. Ohl ICB, Ohl RIB, Chavaglia SRR, Goldman RE. Public actions for control of breast cancer in Brazil: integrative review. Rev Bras Enferm 2016; 69(4):793-803.

6. Santos TLC, Azevedo GM. Estadiamento inicial dos casos de câncer de mama e colo do útero em mulheres brasileiras. Rev Bras Ginecol Obstet 2005; 27(11):656660.

7. Renna Junior NL, Silva GA. Late-stage diagnosis of breast cancer in brazil: analysis of data from hospital -based cancer registries (2000-2012). Rev Bras Ginecol Obstet 2018; 40(3):127-136.

8. Rezende MCR. Causas do diagnóstico tardio no câncer de mama [dissertação]. Rio de Janeiro: Universidade Federal do Rio de Janeiro; 2010.

9. Felix JD, Castro DS, Amorim MHC, Zandonade E. Tendência da mortalidade por câncer de mama em mulheres no estado do Espírito Santo, no período de 1980 a 2007. Rev Bras Cancerol 2011; 57(2):159-166.

10. Lee BL, Liedke PER, Barrios CH, Simon SD, Finkelstein DM, Goss PE. Breast cancer in Brazil: present status and future goals. Lancet Oncol 2012; 13(3):e95-e102.

11. Jacobellis J, Cutter G. Mammography screening and differences in stage of disease by race/ethnicity. Am J Public Health 2002; 92(7):1144-1150.

12. Hahn KM, Bondy ML, Selvan M, Lund MJ, Liff JM, Flagg EW, Brinton LA, Porter P, Eley JW, Coates RJ. Factors associated with advanced disease stage at diagnosis in a population-based study of patients with newly diagnosed breast cancer. Am J Epidemiol 2007; 166(9):1035-1044.

13. Verma R, Bowen RL, Slater SE, Mihaimeed F, Jones JL. Pathological and epidemiological factors associated with advanced stage at diagnosis of breast cancer. Br Med Bull 2012; 103(1):129-145.

14. Rezende MC, Koch HA, Figueiredo JA, Thuler LCS. Factors leading to delay in obtaining definitive diagnosis of suspicious lesions for breast cancer in a dedicated health unit in Rio de Janeiro. Rev Bras Ginecol Obstet 2009; 31(2):75-81.

15. Barry J, Breen N, Barrett M. Significance of increasing poverty levels for determining late-stage breast cancer diagnosis in 1990 and 2000. J Urban Health 2012; 89(4):614-627.

16. Wang F, McLafferty S, Escamilla V, Luo L. Late-stage breast cancer diagnosis and health care access in Illinois. Prof Georg 2008; 60(1):54-69.
17. Trufelli DC, Miranda VC, Santos MBB, Fraile NMP, Pecoroni PG, Gonzaga SFR, Riechelmann Rachel, Kaliks R, Del Giglio A. Análise de atrasos no diagnóstico e tratamento de pacientes com câncer de mama em um hospital público. Rev Assoc Med Bras 2008; 54(1):72-76.

18. Stapleton JM, Mullan PB, Dey S, Hablas A, Gaafar R, Seifeldin IA, Banerjee M, Soliman AS. Patient-mediated factors predicting early- and late-stage presentation of breast cancer in Egypt. Psychooncology 2011; 20(5):532-7.

19. Olivotto IA, Gomi A, Bancej C, Brisson J, Tonita J, Kan L, Mah Z, Harrison M, Shumak R. Influence of delay to diagnosis on prognostic indicators of screen-detected breast carcinoma. Cancer 2002; 94(8):2143-2150

20. Richards MA, Westcombe AM, Love SB, Littlejohns $\mathrm{P}$, Ramirez AJ. Influence of delay on survival in patients with breast cancer: a systematic review. Lancet 1999; 353(9159):1119-1126.

21. Organização Mundial da Saúde. Classificação Estatística Internacional de Doenças e Problemas Relacionados à Saúde. São Paulo: Editora USP; 2012.

22. Organização Mundial de Saúde (OMS). CID-O Classificação Internacional de Doenças para Oncologia. São Paulo: Editora USP; 2013.

23. 5/08 - Mudanças no procedimento de primeira avaliação no INCA. Intranet INCA 2013 Ago 07; Seção Últimas Notícias.

24. Abrahão KS, Bergmann A, Aguiar SS, Thuler LCS. Determinants of advanced stage presentation of breast cancer in 87,969 Brazilian women. Maturitas 2015; 82(4):365-370.

25. Henry KA, Boscoe FP, Johnson CJ, Goldberg DW, Sherman R, Cockburn M. Breast cancer stage at diagnosis: is travel time important? J Community Health 2011; 36(6):933-942.

26. Newman LA. Epidemiology of locally advanced breast cancer. Semin Radiat Oncol 2009; 19(4):195-203.

27. Dos-Santos-Silva I, De Stavola BL, Renna NL Junior, Nogueira MC, Aquino EML, Bustamante-Teixeira MT, Azevedo E Silva G. Ethnoracial and social trends in breast câncer staging at diagnosis in Brazil, 200114: a case only analysis. The Lancet Glob Health 2019; 7(6):e784-e797.

28. Møller H, Henson K, Lüchtenborg M, Broggio J, Charman J, Coupland VH, Davies E, Jack RH, Sullivan R, Vedsted P, Horgan K, Pearce N, Purushotham A. Short-term breast cancer survival in relation to ethnicity, stage, grade and receptor status: national cohort study in England. Br J Cancer 2016; 115(11):1408-1415.

29. Bairros FS, Meneghel SN, Olinto MTA. Citopatológico e exame de mama: desigualdade de acesso para mulheres negras no sul do Brasil. Epidemiol Serv Saúde 2008; 17(2):138-141.

30. Souza EHA, Oliveira PAP, Paegle AC, Goes PSA. Raça e o uso dos serviços de saúde bucal por idosos. Cien Saude Colet 2012; 17(8):2063-2070.

31. Nogueira MC, Maximiliano RG, Cintra JRD, Corrêa CSL, Fayer VA, Bustamante-Teixeira MT. Disparidade racial na sobrevivência em 10 anos para o câncer de mama: uma análise de mediação usando abordagem de respostas potenciais. Cad Saude Publica 2018; 34(9):e00211717. 
32. Brasil. Ministério da Saúde (MS). Política nacional de saúde integral da população negra: uma política para o SUS. Brasília: MS; 2017.

33. Buss PM. Promoção da saúde e qualidade de vida. Cien Saude Colet 2000; 5(1):163-177.

34. Azevedo AO. Análise do IDH do estado do Rio de Janeiro [dissertação]. Rio de Janeiro: Universidade Federal Rural do Rio de Janeiro; 2011.

35. Lannin DR, Mathews HF, Mitchell J, Swanson MS, Swanson FH, Edwards MS. Influence of socioeconomic and cultural factors on racial differences in late-stage presentation of breast cancer. JAMA 1998; 279(22):1801-1807.

36. Osborne C, Ostir GV, Du X, Peek MK, Goodwin JS. The influence of marital status on the stage at diagnosis, treatment, and survival of older women with breast cancer. Breast Cancer Res Treat 2005; 93(1):41-47.

37. Martínez ME, Unkart JT, Tao L, Kroenke CH, Schwab R, Komenaka I, Gomez SL. Prognostic significance of marital status in breast cancer survival: a population -based study. PLoS One 2017; 12(5):e0175515.

38. Palmer MK, Lythgoe JP, Smith A. Prognostic factors in breast cancer. Br J Surg 1982; 69(12):697-698.

39. Ramirez AJ, Westcombe AM, Burgess CC, Sutton S, Littlejohns P, Richards MA. Factors predicting delayed presentation of symptomatic breast cancer: a systematic review. Lancet 1999; 353(9159):1127-1131.

40. Schone BS, Weinick RM. Health related behaviors and the benefits of marriage for elderly persons. Gerontologist 1998; 38(5):618-627.

41. Umberson D. Gender, marital status and the social control of health behavior. Soc Sci Med 1992; 34(8):907-917.

42. Pinquart M, Duberstein PR. Associations of social networks with cancer mortality: a meta-analysis. Crit Rev Oncol Hematol 2010; 75(2):122-137.

43. Kroenke CH, Kubzansky LD, Schernhammer ES, Holmes MD, Kawachi I. Social networks, social support, and survival after breast cancer diagnosis. J Clin Oncol 2006; 24(7):1106-1111.

44. Kroenke CH, Quesenberry C, Kwan ML, Sweeney C, Castillo A, Caan BJ. Social networks, social support, and burden in relationships, and mortality after breast cancer diagnosis in the Life After Breast Cancer Epidemiology (LACE) study. Breast Cancer Res Treat 2013; 137(1):261-271.

45. Chou AF, Stewart SL, Wild RC, Bloom JR. Social support and survival in young women with breast carcinoma. Psychooncology 2012; 21(2):125-133.

46. Epplein M, Zheng Y, Zheng W, Chen Z, Gu K, Penson D, Lu W, Shu XO. Quality of life after breast cancer diagnosis and survival. J Clin Oncol 2011; 29(4):406412.

47. Silva PF. Perfil de mulheres com câncer de mama atendidas em Vitória-ES: influência das variáveis sociodemográficas com o estadiamento clínico do tumor antes do tratamento [dissertação]. Vitória: Universidade Federal do Espírito Santo; 2009.

48. Instituto Nacional de Câncer José Alencar Gomes da Silva (INCA). Falando sobre câncer de mama. Rio de Janeiro: INCA; 2002.
49. Silva EF. Metodologia feminista e direitos reprodutivos no Centro de Saúde Santa Rosa, Niterói (RJ). Saúde Debate 2015; 39(106):893-903.

50. Soares PBM, Quirino Filho S, Souza WP, Gonçalves RCR, Martelli DRB, Silveira MF, Martelli Júnior H. Características das mulheres com câncer de mama assistidas em serviços de referência do Norte de Minas Gerais. Rev Bras Epidemiol 2012; 15(3):595-604.

51. Bertoni N, de Souza MC, Crocamo S, Szklo M, Almeida LM. Is a family history of the breast cancer related to women's cancer prevention behaviors? Int J Behav Med 2019; 26(1):85-90.

52. Mandelblatt J, Andrews H, Kerner J, Zauber A, Burnett W. Determinants of late-stage diagnosis of breast and cervical cancer: the impact of age, race, social class, and hospital type. Am J Publ Health 1991; 81(5):646-649.

53. Wiesner C. Psychological, social and clinical determinants of breast cancer early detection in Bogotá, Colombia. Rev Colomb Cancerol 2007; 11(1):13-22.

54. Silva PF, Amorim MHC, Zandonade E, Viana KCG. Associação entre variáveis sociodemográficas e estadiamento clínico avançado das neoplasias de mama em hospital de referência no estado do Espírito Santo. Rev Bras Cancerol 2013; 59(3):361-367.

55. Olinto MTA, Olinto BA. Raça e desigualdade entre as mulheres: um exemplo no sul do Brasil. Cad Saude Publica 2000; 16(4):1137-1142.

56. Cruz ICF. Saúde e iniquidades raciais no Brasil: o caso da população negra. Online Braz J Nurs 2006; 5(2):216-230.

57. Instituto Nacional de Câncer José Alencar Gomes da Silva (INCA). Ações de enfermagem para o controle do câncer: uma proposta de integração ensino-serviço. Rio de Janeiro: INCA; 2008.

58. Barros AF, Murta-Nascimento C, Abdon CH, Nogueira DN, Lopes ELC, Dias A. Factors associated with time interval between the onset of symptoms and first medical visit in women with breast cancer. Cad Saude Publica 2020; 36(2):e00011919.

59. Nunes LN, Klück MM, Fachel JMG. Uso da imputação múltipla de dados faltantes: uma simulação utilizando dados epidemiológicos. Cad Saude Publica 2009; 25(2):268-278.

Artigo apresentado em 26/03/2020

Aprovado em 01/12/2020

Versão final apresentada em 03/12/2020

Editores-chefes: Romeu Gomes, Antônio Augusto Moura da Silva 